\title{
Some Applications of Orderly Spanning Trees in Graph Drawing*
}

\author{
Ho-Lin Chen ${ }^{1}$, Chien-Chih Liao ${ }^{1}$, Hsueh-I Lu ${ }^{2}$, and Hsu-Chun Yen ${ }^{1}$ \\ 1 Department of Electrical Engineering, National Taiwan University \\ Taipei 106, Taiwan, Republic of China \\ hlchen89@yahoo.com.tw, henry@cobra.ee.ntu.edu.tw \\ yen@cc.ee.ntu.edu.tw, http://www.ee.ntu.edu.tw/ yen/ \\ ${ }^{2}$ Institute of Information Science, Academia Sinica \\ Taipei 115, Taiwan, Republic of China \\ hil@iis.sinica.edu.tw, http://www.iis.sinica.edu.tw/ ${ }^{\text {hil/ }}$
}

\begin{abstract}
Orderly spanning trees seem to have the potential of becoming a new and promising technique capable of unifying known results as well as deriving new results in graph drawing. Our exploration in this paper provides new evidence to demonstrate such a potential. Two applications of the orderly spanning trees of plane graphs are investigated. Our first application deals with Podevs drawing, i.e., planar orthogonal drawing with equal vertex size, introduced by Fößmeier and Kaufmann. Based upon orderly spanning trees, we give an algorithm that produces a Podevs drawing with half-perimeter no more than $\left\lceil\frac{3 n}{2}\right\rceil+1$ and at most one bend per edge for any $n$-node plane graph with maximal degree $\Delta$, a notable improvement over the existing results in the literature in terms of the size of the drawing area. The second application is an alternative proof for the sufficient and necessary condition for a graph to admit a rectangular dual, i.e., a floor-plan using only rectangles.
\end{abstract}

\section{Introduction}

Graph drawing has emerged as an exciting and fast growing area of research in the computer science community in recent years [10]. Among various techniques for drawing planar graphs, the canonical orderings of triconnected plane graphs have served as a fundamental step upon which many drawing algorithms are built $[6,9,12,15,16,18,19]$. The work by de Fraysseix, Pach and Pollack [9] is considered to be the first using the canonical orderings to produce straight-line drawings with polynomial sizes for planar graphs. The technique of canonical orderings has subsequently been applied to drawing graphs with respect to a variety of aesthetic constraints, including straight-line, convexity, orthogonality, 2-visibility, floor-planning, among others. Alternatively, Schnyder [32] introduced the concept of realizers which provides an interesting characterization for vertices and edges in triangulated planar graphs. Applications of realizers to graph drawing can be found in $[8,32]$, for instance.

\footnotetext{
* Research supported in part by NSC Grant 90-2213-E-002-100.
} 


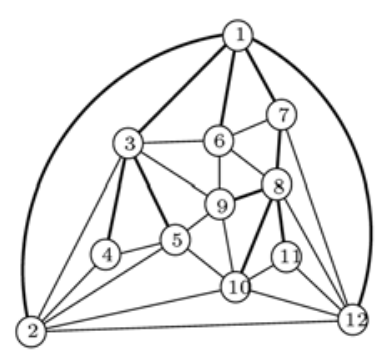

(a)

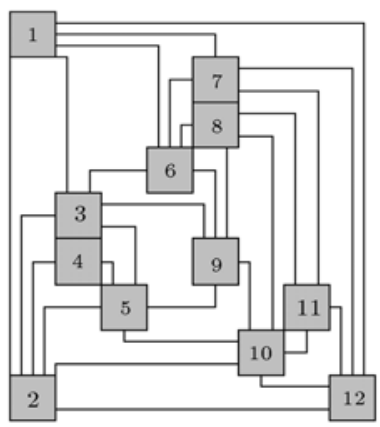

(b)

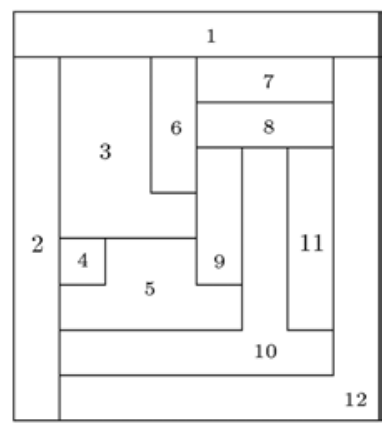

(c)

Fig. 1. (a) A plane triangulation $G$, where an orderly spanning tree $T$ of $G$ rooted at node 1 is drawn in dark. The node labels show the counterclockwise preordering of the nodes in $T$. (b) A Podevs drawing of $G$. (c) A floor-plan of $G$.

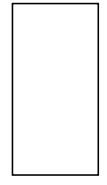

(a)

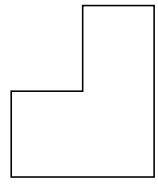

(b)

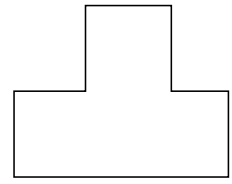

(c)

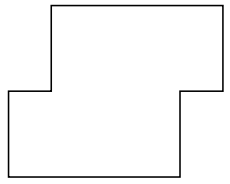

(d)

Fig. 2. Module types: (a) I-module, (b) L-module, (c) T-module, and (d) Z-module.

Recently, Chiang, Lin, Lu [5] introduced the concept of orderly spanning trees for planar graphs, extending that of canonical ordering for plane graphs not required to be triconnected and that of realizer for plane graphs not required to be triangulated. In fact, for a plane triangulation $G$, (i) if $T$ is an orderly spanning tree of $G$, then the counterclockwise preordering of the nodes of $T$ is always a canonical ordering of $G$, and (ii) if $\left(T_{1}, T_{2}, T_{n}\right)$ is a realizer of $G$, where $T_{i}$ is rooted at $v_{i}$ for each $i=1,2, n$, then each $T_{i}$ plus both external edges of $G$ incident to $v_{i}$ is an orderly spanning tree of $G$. In addition, orderly spanning trees of planar graphs can be computed in linear time [5]. In light of their recent success in establishing floor-planning [23] and 2-visibility drawing [5], we feel that orderly spanning trees have the potential of becoming a new and promising technique capable of unifying known results as well as deriving new results in graph drawing. Along this line of research, in this article we demonstrate two additional applications of orderly spanning trees in graph drawing, namely, Podevs drawing and rectangular dual. For more about the applications of ordering spanning trees, the interested reader is referred to [5] for succinct graph encoding and [24] for the design of compact routing tables.

Our first application of orderly spanning tree is concerned with Podevs drawings of planar graphs, introduced by Fößmeier and Kaufmann [13]. In Podevs drawings, all vertices are given as identical $k \times k$ squares, with the size $k$ de- 
termined appropriately. Edges are routed as axis-parallel paths along the grid lines. See Figure 1(b) for an illustration. Let $\Delta$ be the degree of the input $n$-node $m$-edge planar graph. Fößmeier and Kaufmann [13] gave a linear-time algorithm for computing a $(\Delta+1) n \times(\Delta+1) n$ Podevs drawing with at most $2 m$ bends. ${ }^{1}$ They also gave an $O\left(n^{2} \log n\right)$-time algorithm, extending from Tamassia's classic result [35], that computes a Podevs drawing with minimum number of bends. ${ }^{2}$ Bonichon, Le Saëc and Mosbah [4] gave a linear-time algorithm to obtain a $(\Delta-1)(n-2) \times(\Delta-1)(n-1)$ Podevs drawing with at most one bend per edge. They also provided a $\left\lfloor\frac{\Delta}{2}\right\rfloor(n-2) \times\left\lfloor\frac{\Delta}{2}\right\rfloor(n-1)$ Podevs drawing with at most $\left\lfloor\frac{3 m}{2}\right\rfloor$ bends. Based upon orderly spanning trees, we give a linear-time algorithm to produce a Podevs drawing with half-perimeter (i.e., height plus width) no more than $\left(\left\lceil\frac{3 n}{2}\right\rceil+1\right) \Delta$ and at most one bend per edge, a notable improvement over $[4,13]$ in terms of the drawing area. ${ }^{3}$

Our second application has to do with floor-planning planar graphs. Floorplanning $[25,36]$, a fundamental step in VLSI chip design, refers to the process of, given a graph whose nodes (respectively, edges) representing functional entities (respectively, interconnections), partitioning a rectangular chip area into a set of non-overlapping rectilinear polygonal modules (each of which describes a functional entity) in such a way that the modules of adjacent nodes share a common boundary. For example, Figure 1(c) is a floor-plan of the graph in Figure 1(a). For floor-planning general plane graphs, Yeap and Sarrafzadeh [37] showed that rectilinear modules with at most two concave corners are sufficient and necessary. He [15], measuring the complexity of a module in terms of the number of its constituent rectangles, presented a linear-time algorithm to construct a floor-plan of a plane triangulation using modules, each of which is a union of two disjoint rectangles (called 2-rectangular modules). More recently, Liao, Lu and Yen [23] used orderly spanning trees to demonstrate a linear-time algorithm to layout, given an $n$-node plane triangulation, a floor-plan in an area bounded by $(n-1) \times\left\lfloor\frac{2 n+1}{3}\right\rfloor$ using three basic modules, namely, I-module, $L$-module, and T-module depicted in Figures 2(a), 2(b), and 2(c), respectively. (He's algorithm [15] may produce a floor-plan containing Z-modules as shown in Figure 2(d).) A floor-plan using only rectangles to represent nodes is called a rectangular dual. It was shown in [20-22] that an internally triangulated plane $G$ admits a rectangular dual if and only if $G$ has four exterior nodes, and $G$ has no

\footnotetext{
1 The area bound mentioned in [13] is $2(\Delta+1) n \times(\Delta+1) n$, which has to do with the $2 n \times n$ straight-line drawing of [7]. Using an $n \times n$ straight-line drawing of $G$ (e.g., [32]), the area bound of [13] can be reduced by a factor of two.

2 The optimality for the number of bends has to do with an additional property required by the drawing. That is, each nontrivial face should occupy a nonempty region in the drawing, which is for putting the labels of faces. Such a drawing is called a Podevsnef drawing, standing for planar orthogonal drawing with equal vertex size and none-empty face. Very recently, Mutzel and Weiskircher [28] resorted to integer programming to minimize the number of bends in such a drawing.

${ }^{3}$ Under slightly modified conditions, e.g., the nodes need not be of the same size, there are efficient algorithms producing more compact orthogonal drawings $[1-3,11$, $12,30]$.
} 
separating triangles. ${ }^{4}$ In this paper, we are able to provide an alternative proof, based upon orderly spanning trees, for the above sufficient and necessary condition for a graph to admit rectangular dual. Aside from offering a simpler proof in comparison with that of $[36,37]$, we feel that our exploration might lead to new algorithms and characterization for the so-called slicible floor-plans [29, 34], i.e., those obtainable by means of horizontal or vertical cuts. As we shall see later, our exploration also leads to a simple way of achieving proper box rectangular drawing of $[16,31]$.

The remainder of this paper is organized as follows. Section 2 gives the preliminaries. Section 3 presents our Podevs drawing algorithm. Section 4 shows the sufficient and necessary condition for a planar graph that admits a rectangular dual. Section 5 concludes the paper with future work and some comments on applying rectangular-dual algorithms to obtaining proper box-rectangular drawing.

\section{Preliminaries}

A plane graph is a planar graph equipped with a fixed planar embedding. The embedding of a plane graph divides the plane into a number of connected regions, each of which is called a face. The unbounded face of $G$ is called the exterior face, whereas the remaining faces are interior faces. $G$ is a plane triangulation if $G$ has at least three nodes and the boundary of each face, including the exterior face, of $G$ is a triangle. Let $T$ be a rooted spanning tree of a plane graph $G$. Two nodes are unrelated in $T$ if they are distinct and neither of them is an ancestor of the other in $T$. An edge of $G$ is unrelated with respect to $T$ if its endpoints are unrelated in $T$. Let $v_{1}, v_{2}, \ldots, v_{n}$ be the counterclockwise preordering of the nodes in $T$. A node $v_{i}$ is orderly in $G$ with respect to $T$ if the neighbors of $v_{i}$ in $G$ form the following four blocks in counterclockwise order around $v_{i}$ :

$B_{1}\left(v_{i}\right)$ : the parent of $v_{i}$,

$B_{2}\left(v_{i}\right)$ : the unrelated neighbors $v_{j}$ of $v_{i}$ with $j<i$,

$B_{3}\left(v_{i}\right)$ : the children of $v_{i}$, and

$B_{4}\left(v_{i}\right)$ : the unrelated neighbors $v_{j}$ of $v_{i}$ with $j>i$,

where each block could be empty. $T$ is an orderly spanning tree of $G$ if $v_{1}$ is on the boundary of $G$ 's exterior face, and each $v_{i}, 1 \leq i \leq n$, is orderly in $G$ with respect to $T$. It is not difficult to see that if $G$ is a plane triangulation, then $B_{2}\left(v_{i}\right)$ (respectively, $B_{4}\left(v_{i}\right)$ ) is nonempty for each $i=3,4, \ldots, n$ (respectively, $i=2,3, \ldots, n-1$. For each $i=2,3, \ldots, n$, let $p(i)$ be the index of the parent of $v_{i}$ in $T$. Let $w(i)$ denote the number of leaves in the subtree of $T$ rooted at $v_{i}$. Let $\ell(i)$ and $r(i)$ be the functions such that $v_{\ell(i)}$ (respectively, $v_{r(i)}$ ) is the last (respectively, first) neighbor of $v_{i}$ in $B_{2}\left(v_{i}\right)$ (respectively, $B_{4}\left(v_{i}\right)$ ) in counterclockwise order around $v_{i}$. For example, in the example shown in Figure 1(a),

\footnotetext{
${ }^{4}$ A separating triangle, which is also known as complex triangle [36,37], is a cycle of three edges enclosing some nodes in its interior. For example, $(2,3,5)$ and $(8,10,12)$ are two separating triangles of the graph shown in Figure 1(a).
} 
one can easily verify that node 3 is indeed orderly with respect to $T$, where $B_{1}(3)=\{1\}, B_{2}(3)=\{2\}, B_{3}(3)=\{4,5\}, B_{4}(3)=\{6,9\}, p(3)=1, w(3)=2$, $\ell(3)=2$, and $r(3)=9$. When $G$ is a plane triangulation, it is known [5] that for each edge $\left(v_{i}, v_{j}\right)$ of $G-T$ with $i<j$, at least one of $i=\ell(i)$ and $j=r(i)$ holds. To be more specific, if $i=2$ and $j=n$, then both $3=\ell(n)$ and $n=r(3)$ hold; otherwise, precisely one of $i=\ell(i)$ and $j=r(i)$ holds. The following lemma is an important property of orderly spanning tree.

Lemma 1 (see [5]). Given an n-node plane triangulation $G$, an orderly spanning tree $T$ of $G$ with at most $\left\lfloor\frac{2 n+1}{3}\right\rfloor$ leaves is obtainable in $O(n)$ time. ${ }^{5}$

A floor-plan $F$ of $G$ is a partition of a rectangle into $n$ non-overlapping rectangular modules $r_{1}, r_{2}, \ldots, r_{n}$ such that $v_{i}$ and $v_{j}$ are adjacent in $G$ if and only if the boundaries of $r_{i}$ and $r_{j}$ share at least one non-degenerated line segment. The size of $F$ is the area of the rectangle being partitioned by $F$ with the convention that the corners of all modules are placed on integral grid points. For example, the size of the floor-plan shown in Figure $1(\mathrm{c})$ is $8 \times 7$. Based upon Lemma 1 , Liao, Lu, and Yen [23] gave a linear-time algorithm for obtaining floor-plans for plane triangulations with the best known worst-case size.

Lemma 2 (see [23]). Given an n-node plane triangulation $G$ with $n \geq 3$, a floor-plan $F$ of $G$ can be constructed in $O(n)$ time such that $F$ consists of $I$ modules, L-modules, and T-modules only. The size of $F$ is at most $(n-1) \times$ $\left\lfloor\frac{2 n+1}{3}\right\rfloor$.

\section{Podevs Drawings}

The following corollary of Lemma 2 shows how to obtain a Podevs drawing from the output floor-plan of [23].

Corollary 1. Let $G$ be an n-node internally triangulated plane graph with at least four vertices on the external boundary of $G$. Let $a$ and $b$ be two nodes of $G$ on the external boundary of $G$. Then, $G$ admits an $(n-1) \Delta \times\left\lfloor\frac{2 n+1}{3}\right\rfloor \Delta$ Podevs drawing with at most one bend per edge. Moreover, nodes a and $b$ on the external cycle have only upward edges except edge $(a, b)$.

Proof. (sketch) We first compute a floor-plan as guaranteed by Lemma 2. Now for each module, we place a unit square at a position that is visible to all points in the module. It is not difficult to see that one can connect the squares corresponding to two adjacent nodes in $G$ by a line with one bend such that no two edges intersect each other. The corollary can be obtained by enlarging the above drawing by a factor of $\Delta$. For example, the Podevs drawing in Figure 1(b) is obtained this way.

${ }^{5}$ Recently, Mosbah, Bonichon, and Le Saëc [26] proved a tighter bound $\left\lfloor\frac{2 n-f+1}{3}\right\rfloor$ on the number of leaves in an orderly spanning tree from the viewpoint of realizers, where $f$ is the number of "cyclic faces" in a realizer of $G$. In the extreme cases, $f$ could be $O(1)$. 
Let $G$ be a plane triangulation on $n$ nodes $v_{1}, v_{2}, \ldots, v_{n-1}, r$, where $r$ belongs to the external boundary of $G$, such that $\left(r, v_{i}\right)$ is an edge of $G$ for each $i=$ $1,2, \ldots, n-1$. Let $G_{a, b}$ denote the subgraph of $G$ induced by $r, v_{a}, v_{a+1}, \ldots, v_{b}$. We have the following two lemmas. Let $h(\Gamma)$ (respectively, $w(\Gamma)$ and $h w(\Gamma)$ ) denote the number $x$ such that $x \Delta$ is the required height (respectively, width and half-perimeter) of the drawing $\Gamma$.

Lemma 3. G has a Podevs drawing $\Gamma$ with $w(\Gamma) \leq(n-1), h(\Gamma) \leq\left\lfloor\frac{n}{2}\right\rfloor+1$ that has at most one bend per edge. Moreover, if $n$ is odd, then nodes $r, v_{1}, v_{n-1}$ and edge $\left(r, v_{n-1}\right)$ occupy the four corners of $\Gamma$; otherwise, nodes $r, v_{1}$, edge $\left(v_{1}, v_{n-1}\right)$, and edge $\left(v_{n-1}, r\right)$ occupy the four corners of $\Gamma$.

Proof. (sketch) We prove the lemma by induction on $n$. Clearly, the lemma holds trivially for $n=4$ and $n=5$. As for the induction step, consider the case that $k$ is odd. We first find the index $i$ such that $v_{i}$ is connected to both $v_{1}$ and $v_{k-1}$. If $i \neq 2$ or $i \neq k-2$, then we may assume without loss of generality that $i$ is odd. By the inductive hypothesis, we can obtain drawings of $G_{1, i}$ and $G_{i, n-1}$ with total width no more than $k-1$ whose maximum height is at most $\left\lfloor\frac{k}{2}\right\rfloor$. Now we combine the graphs to get the drawing of $G$, as shown in Figure 3(a). If $i=2$, then we can draw $G_{i, k-1}$ and add $v-1$ nodes properly, each increasing the width by one, to get $\Gamma$, as shown in Figure 3(b). The case $i=k-2$ is similar.

The case that $k$ is even can be proved similarly.

Lemma 4. $G_{a, b}$ has a Podevs drawing $\Gamma$ with $h w(\Gamma) \leq(n-1)+\left\lceil\frac{n}{2}\right\rceil+1$ that contains at most one bend per edge. The other two nodes $a$ and $b$ on the external cycle has only upward edges except edge $(a, b)$ and are on consecutive corners of the drawing.

Proof. (sketch) If $i \neq 2$ or $i \neq k-2$, find the node $v_{i}$ such that $v_{i}$ is adjacent to all three nodes on the external boundary. Without loss of generality, we may assume $i \geq(k-1) / 2$. By Corollary 1 , we can find a drawing $D_{1}$ of $G_{i, k-2}$ with $h\left(D_{1}\right) \leq k-i$ and $w\left(D_{1}\right) \leq k-i$ such that $r$ and $v_{i}$ are on two consecutive corners. Let $j \neq 1$ be the smallest number such that $v_{i}$ and $v_{j}$ are connected. By Lemma 3 , we have a drawing $D_{2}$ of $G_{j, i}$ with $w\left(D_{2}\right) \leq i-j+1$ and $h\left(D_{2}\right) \leq\left\lfloor\frac{i-j}{2}\right\rfloor+2$ such that $r, v_{j}, v_{i}$ are three consecutive corners of $D_{2}$. Combining the two drawings and adjust the position of $r, v_{i}$, and $v_{1}$ properly, we can get a drawing $D$ of $G$ with $h w(D) \leq k-i+\left\lfloor\frac{i-j}{2}\right\rfloor+1+i-j+1+j \leq k+\left\lfloor\frac{i-j}{2}\right\rfloor+2 \leq(k-1)+\left\lfloor\frac{k}{2}\right\rfloor+2$. The second equality holds only when $j=2$ and the third equality holds only when $i=k-2$. If $i \neq k-2$ or $j \neq 2$, then the $h w(D) \leq(k-1)+\left\lfloor\frac{k}{2}\right\rfloor+1$. If $i=k-2$ and $j=2$, then by Lemma 3 we can draw $G_{j, i}$ in a $(k-3) \times\left\lfloor\frac{k}{2}\right\rfloor$ area. If $k$ is even, then we can add $v_{1}$ and $v_{i}$ such that the width and height of the drawing are increased by 2 and 1 , respectively. If $k$ is odd, then we can add $v_{1}$ and $v_{i}$ such that the width and height of the drawing are both increased by 2 . In both cases, we have $h w(D) \leq(k-1)+\left\lceil\frac{k}{2}\right\rceil+1$.

Theorem 1. Any n-node plane graph $G$ has a Podevs drawing with half-perimeter no more than $\left(\left\lceil\frac{3 n}{2}\right\rceil+1\right)(\Delta-1)$ and width no more than $\left(\left\lfloor\frac{2 n}{3}\right\rfloor+1\right)(\Delta-1)$. 

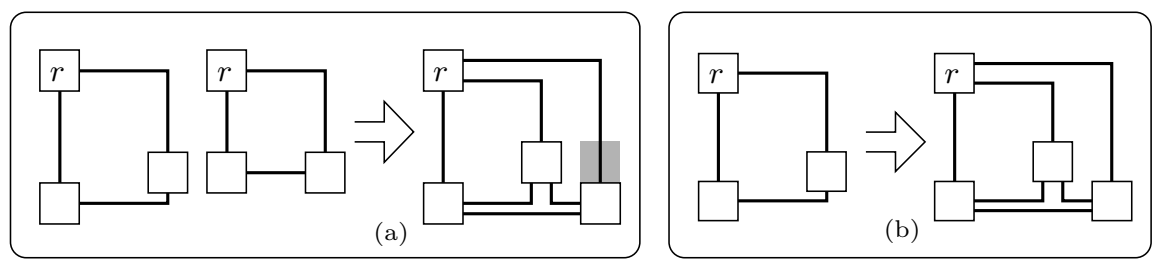

Fig. 3. (a) The left drawing is for $G_{1, i}$, the middle drawing is for $G_{i, n-1}$, and the right drawing is the combined one. (b) The left drawing is for $G_{1, n-2}$ and the right drawing is the resulting drawing of adding one node.

Proof. (sketch) Find an orderly spanning tree $T$ of $G$. Let the number of leaves of $T$ be $m$. A cluster of leaves in $T$ is a maximum set of connected leaves of $T$ with the same parent in $T$. If $m \leq n / 2$, then the drawing obtained from the original floor-plan satisfies the requirement. Otherwise, by Corollary 1, we can get a drawing $\Gamma$ of $G$ with $w(\Gamma) \leq m$ and $h(\Gamma) \leq n-1$. Using Lemma 4, for each cluster of the leaves, we can adjust the drawing $\Gamma$ such that a cluster of $t$ leaves in an area with half-perimeter bounded by $(t+3-1)+\left\lceil\frac{t+3}{2}\right\rceil+1-4=t+\left\lfloor\frac{t}{2}\right\rfloor+1$. The half-perimeter of the drawing is at most $n-m / 2+m+N_{e}+N_{o} / 2$, where $N_{e}$ is the number of clusters with even number of leaves and $N_{o}$ is the number of clusters with odd number of leaves.

For any two clusters $C_{1}$ and $C_{2}$ of leaves, we say that $C_{1}$ is an ancestor of $C_{2}$ if an ancestor of $C_{2}$ is connected to some leaves in $C_{1}$. If $C_{1}$ is an ancestor of $C_{2}$ and the number of leaves in $C_{2}$ is more than one, then let the parent of $C_{2}$ be $N_{2}$, we can reduce the width by 1 by shifting the subtree rooted at $N_{2}$. If for two clusters $C_{i}$ and $C_{j}$, we cannot find a sequence of clusters $C_{i}, C_{i+1}, \cdots, C_{j}$ such that $C_{a}$ is an ancestor of $C_{a+1}$, for all $a$, then the height of the drawing can be reduced by 1 since the two clusters may share the same level of the Podevs drawing. Suppose the sequence of clusters that has the maximum height is $S$. We know that the half-perimeter of the drawing is at most $n-m / 2+m+N_{e}(S)+N_{o}(S) / 2-N_{t}(S) \leq$ $\left\lceil\frac{3 n}{2}\right\rceil+1$, where $N_{e}(S)$ is the number of clusters in $S$ that has even number of leaves, $N_{o}(S)$ is the number of clusters in $S$ that has odd number of leaves, and $N_{t}(S)$ is the number of clusters in $S$ that has at least two leaves. Since the width of the drawing in Lemma 3 is at most $n-1$. The above operations do not increase the width of the drawing. Therefore, the width of the drawing is at most $m$. By Lemma 2, we can find the desired drawing of the graph.

Now we expand each node by the same factor. One problem occurs when there is a straight edge connecting two nodes such that two nodes cannot put in the same level, see Figure 4. We define a new notation: for a node $N$ in the graph, edge-right-bend-up $(N)$ is the number of edges attach to the right-hand side of the node $N$ and have an upward bend. (Similar for others.) The rest of the proof shows that The graph can be properly rearranged such that the node size is smaller than $(\Delta-1) \times(\Delta-1)$.

The only problem occurs when there is a straight edge connecting two nodes. According to our algorithm, one node will have more than one downward edges 

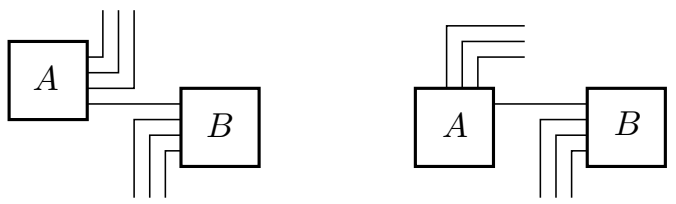

Fig. 4.

if and only if its children are leaves which have already been rearranged. In this case, there is no downward straight edge. Otherwise, the node has only one downward edge and can be attached to any point of the node. So, we can always arrange the downward straight edge to satisfy the constraint that the node size is smaller than $(\Delta-1) \times(\Delta-1)$.

Consider the case where two nodes $A$ and $B$ are connected by a horizontal straight edge and edge-right-bend-up $(A)+1+$ edge-left-bend-down $(B) \geq \Delta-1$. (See Figure 4). Rearrange edge-right-bend-up $(A)+$ edge-left-bend-down $(B)-\Delta+1$ of the nodes connecting to $A$ such that the edges are connected to the upward side of the node $A$ and bends toward right. (As shown in Figure 4.) This change may increase the height of the graph at most edge-right-bend-up $(A)$ but can always be compensated since there are edge-right-bend-up $(A)$ nodes connecting to $A$ and edge-left-bend-down $(B)$ nodes connecting to $B$ that can share columns and hence decrease the width of the graph by the minimum of edge-right-bend-up $(A)$ and edge-left-bend-down $(B)$.

\section{Rectangular Dual}

This section gives an alternative proof for the following theorem.

Theorem 2. Every internally triangulated plane graph $G$ without complex triangle has a rectangular dual if and only if $G$ has at least four vertices on the external boundary of $G$.

For a internally triangulated plane graph $G$ and a node $r$ and at least three other nodes on the external cycle, let floor-plan $(G, r)$ be a floor-plan of $G$ consisting of I-modules, L-modules, and T-modules such that $r$ completely occupies one of its four sides. By Lemma 2, such a floor-plan can be obtained in linear time. In such a floor-plan, the level of a node $v$, denoted $\ell(v)$, is defined as the distance between the bottom boundaries of $r$ and $v$. For example, we have $\ell(1)=0, \ell(7)=1, \ell(8)=2$, and $\ell(5)=6$ in the floor-plan shown in Figure 1(c).

Lemma 5. If a plane graph $G$ is internally triangulated and has no complex triangle, then for any three consecutive nodes $a, r, b$ on the external cycle of $G$, there is a rectangular dual of $G$ with $a, r, b$ completely occupying three sides of the drawing.

Proof. (sketch) We prove the theorem by induction on the number of nodes in $G$. Clearly, the theorem holds trivially if $G$ has at most three nodes. Suppose 

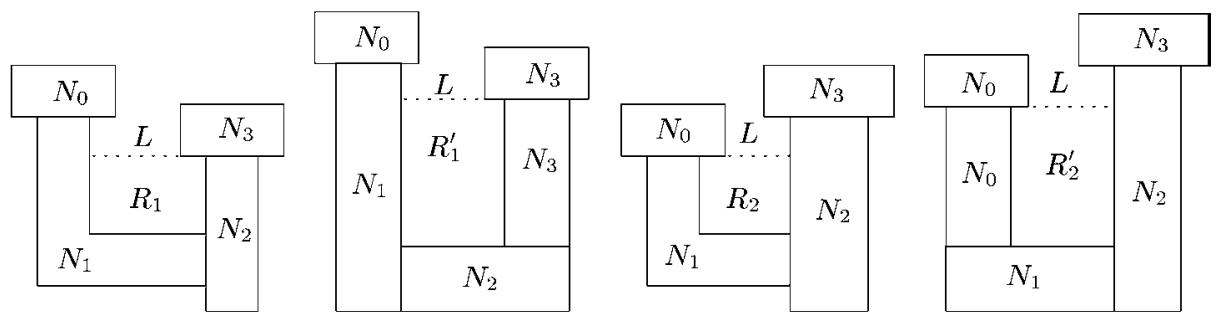

Fig. 5. An illustration for our algorithm for rectangular dual.

for any plane graph $G$ with less than $n$ nodes, the theorem holds. For a graph $G$ with $n$ nodes and nodes $a, r, b$ specified on the external cycle, we can find a rectangular dual by the following process.

Step 1. Triangulate $G$ so that $(a, r, b)$ is the external boundary of the triangulated graph.

Step 2. By computing floor-plan $(G, r)$, we get a floor-plan $F$ of $G$ consisting of only L-modules and T-modules. We then repeat the following three steps until all nodes are picked and processed in Step 3.

Step 3. Choose the node $N_{1}$ having the lowest level among all L-module and T-module nodes. Without loss of generality, we may assume that $N_{1}$ bends to the right, the node at the right-hand side of $N_{1}$ is $N_{2}$, and the parents of $N_{1}, N_{2}$ are $N_{0}, N_{3}$, respectively, as shown in Figure 5(a).

Step 4. If $\ell\left(N_{0}\right)<\ell\left(N_{3}\right)$, then draw a horizontal line $L$ at the bottom of $N_{3}$. Let $G_{1}$ be the subgraph of $G$ induced by all nodes surrounded by $N_{1}$, $N_{2}, N_{3}$ and $L$. By the induction hypothesis, we can find a rectangular dual $R_{1}$ of $G_{1}$ such that $N_{1}, N_{2}, N_{3}$ completely occupy the remaining three sides of $R_{1}$. Adjust $R_{1}$ such that the four corners of $R_{1}$ are $N_{1}$, $N_{1}, N_{2}$, and $N_{3}$ respectively. Expand $R_{1}$ and $F$ so that all the nodes on the external cycle of $R_{1}$ have the same size in two graphs. Replace the subgraph $G_{1}$ in $F$ by expanding $R_{1}$.

Step 5. If $\ell\left(N_{3}\right)<\ell\left(N_{0}\right)$, then draw a horizontal line $L$ at the bottom of $N_{0}$. Let $G_{2}$ be the subgraph of $G$ induced by all nodes surrounded by $N_{0}$, $N_{1}, N_{2}$ and $L$. By the inductive hypothesis, we can find a rectangular dual $R_{2}$ of $G_{2}$ such that $N_{0}, N_{1}, N_{2}$ are three sides of $R_{2}$. Adjust $R_{2}$ such that the four corners of $R_{2}$ belong to $N_{0}, N_{1}, N_{2}, N_{2}$, respectively. Expand $R_{2}$ and $F$ so that all the nodes on the external cycle of $R_{2}$ have the same size in two graphs. Replace the subgraph $G_{2}$ in $F$ by expanding $R_{2}$.

Since we maintain the property stated in Step 2 and the number of bends is reduced by 1 each time, we can get a rectangular dual of $G$ in $O(n)$ iterations. 

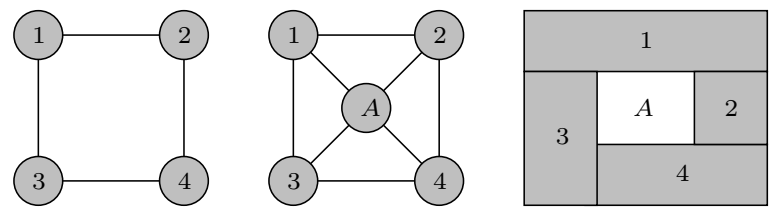

Fig. 6. An illustration for using rectangular duals to obtain proper box rectangular drawings.

\section{Concluding Remarks}

In conventional floor-planning, two rectangles are may be adjacent even if the two modules corresponding to them do not have interconnections. Such unwanted adjacencies are not desirable in some other floor-planning problem. In floorplanning of a Multi-chip Module (MCM), two chips generating excessive heat should not be adjacent, or two chips operating on high frequency should not be adjacent to avoid malfunctioning due to their interference [33]. Unwanted adjacencies may cause a dangerous situation in some architectural floor-planning, too $[14,16,31]$. A box-rectangular drawing [31] has the nice property of avoiding unwanted adjacency of modules, but may draw some vertices as points. A proper box rectangular drawing, introduced by He [16], requires every vertex to be drawn as a real box.

A rectangular-dual algorithm gives an alternative way for obtaining a proper box rectangular drawing. First of all, we add a new vertex $v$ into each nontriangular internal face $F$ of the given biconnected graph $G$ with at least four nodes on the external boundary. We then triangulate $F$ by adding an edge from $v$ to each node on the boundary of $F$. $G$ becomes a triangular plane graph $G^{\prime}$. We then obtain a rectangular dual $D^{\prime}$ for $G^{\prime}$. A proper box rectangular drawing $D$ of $G$ can be obtained from $D^{\prime}$ by turning the module for each newly introduced vertex into a dead space. Clearly, two rectangles are always adjacent if and only if the two modules corresponding to them have interconnections in $G$ and $G^{\prime}$. See Figure 6 for an example.

An interesting possibility for future work is to see whether orderly spanning trees play any constructive role in characterizing slicible floor-plans. It would also be interesting to close the gap between the upper and lower bounds for Podevs drawing for planar graphs, and to see whether new results concerning Podevs drawing with two or more bends can be derived.

\section{References}

1. P. Bertolazzi, G. Di Battista, and W. Didimo. Computing orthogonal drawings with the minimum number of bends. IEEE Transactions on Computers, 49(8):826$840,2000$.

2. T. Biedl and M. Kaufmann. Area-efficient static and incremental graph drawings. In Proceedings of the 5th European Symposium on Algorithms, Lecture Notes in Computer Science 1284, pages 37-52. Springer-Verlag, 1997. 
3. T. Biedl, B. Madden, and I. Tollis. The three-phase method: A unified approach to orthogonal graph drawing. International Journal of Computational Geometry and Applications, 10(6):553-580, 2000.

4. N. Bonichon, B. Le Saëc, and M. Mosbah. Orthogonal drawings based on the stratification of planar graphs. In Proceedings of the 6th International Conference on Graph Theory, Electronic Notes in Discrete Math 5, Marseille, France, 2000. Elsevier. A full version can be found at http://citeseer.nj.nec.com/bonichon00orthogonal.html.

5. Y.-T. Chiang, C.-C. Lin, and H.-I. Lu. Orderly spanning trees with applications to graph encoding and graph drawing. In Proceedings of the 12th Annual ACM-SIAM Symposium on Discrete Algorithms, pages 506-515, Washington, D. C., USA, 7-9 Jan. 2001. A revised and extended version can be found at http://xxx.lanl.gov/abs/cs.DS/0102006.

6. M. Chrobak and G. Kant. Convex grid drawings of 3-connected planar graphs. International Journal of Computational Geometry \& Applications, 7(3):211-223, 1997.

7. M. Chrobak and T. H. Payne. A linear-time algorithm for drawing a planar graph on a grid. Information Processing Letters, 54(4):241-246, May 1995.

8. H. de Fraysseix, P. Ossona de Mendez, and P. Rosenstiehl. On triangle contact graphs. Combinatorics, Probability and Computing, 3:233-246, 1994.

9. H. de Fraysseix, J. Pach, and R. Pollack. How to draw a planar graph on a grid. Combinatorica, 10:41-51, 1990.

10. G. di Battista, P. Eades, R. Tammassia, and I. Tollis. Graph Drawing: Algorithms for the Visualization of Graphs. Prentice Hall, 1998.

11. M. Eiglsperger and M. Kaufmann. Fast compaction for orthogonal drawings with vertices of prescribed size. In Mutzel et al. [27], pages 124-138.

12. U. Fößmeier, G. Kant, and M. Kaufmann. 2-visibility drawings of planar graphs. In S. North, editor, Proceedings of the 4th International Symposium on Graph Drawing, Lecture Notes in Computer Science 1190, pages 155-168, California, USA, 1996. Springer-Verlag.

13. U. Fößmeier and M. Kaufmann. Drawing high degree graphs with low bend numbers. In S. Whitesides, editor, Proceedings of the 3th International Symposium on Graph Drawing, Lecture Notes in Computer Science 1027, pages 254-266, Passau, Germany, 1995. Springer-Verlag. A full version: Technical Report WSI-95-21, Wilhelm-Schickard-Institut Universität Tübingen, 1995.

14. R. L. Francis and J. A. White. Facility layout and location. Prentice-Hall, New Jersey, 1974.

15. X. He. On floor-plan of plane graphs. SIAM Journal on Computing, 28(6):21502167, 1999.

16. X. He. A simple linear time algorithm for proper box rectangular drawings of plane graphs. Journal of Algorithms, 40(1):82-101, 2001.

17. O. H. Ibarra and L. Zhang, editors. Proceedings of the 8th International Conference on Computing and Combinatorics, Lecture Notes in Computer Science 2387, Singapore, August 15-17 2002. Springer.

18. G. Kant. Drawing planar graphs using the canonical ordering. Algorithmica, 16(1):4-32, 1996.

19. G. Kant and X. He. Regular edge labeling of 4-connected plane graphs and its applications in graph drawing problems. Theoretical Computer Science, 172(12):175-193, 1997.

20. K. Koźmiński and E. Kinnen. Rectangular duals of planar graphs. Networks, 15(2):145-157, 1985. 
21. K. A. Kózmiński and E. Kinnen. Rectangular dualization and rectangular dissections. IEEE Transactions on Circuits and Systems, 35(11):1401-1416, 1988.

22. Y. T. Lai and S. M. Leinwand. A theory of rectangular dual graphs. Algorithmica, $5(4): 467-483,1990$.

23. C.-C. Liao, H.-I. Lu, and H.-C. Yen. Floor-planning via orderly spanning trees. In Mutzel et al. [27], pages 367-377.

24. H.-I. Lu. Improved compact routing tables for planar networks via orderly spanning trees. In Ibarra and Zhang [17], pages 57-66.

25. K. Mailing, S. H. Mueller, and W. R. Heller. On finding most optimal rectangular package plans. In Proceedings of the 19th Annual IEEE Design Automation Conference, pages 263-270, 1982.

26. M. Mosbah, N. Bonichon, and B. Le Saëc. Wagner's theorem on realizers. In M. Hennessy and P. Widmayer, editors, Proceedings of the 29th International Colloquium on Automata, Languages, and Programming, Lecture Notes in Computer Science 1443, pages 1043-1063, Málaga, Spain, 2002. Springer-Verlag.

27. P. Mutzel, M. Jünger, and S. Leipert, editors. Proceedings of the 9th International Symposium on Graph Drawing, Lecture Notes in Computer Science 2265, Vienna, Austria, 2001. Springer.

28. P. Mutzel and R. Weiskircher. Bend minimization in orthogonal drawings using integer programming. In Ibarra and Zhang [17], pages 484-493.

29. R. Otten. Efficient floorplan optimization. In Proceedings of International Conference on Computer Design, pages 499-503, Port Chester, New York, 1983.

30. A. Papakostas and I. G. Tollis. Efficient orthogonal drawings of high degree graphs. Algorithmica, 26(1):100-125, 2000.

31. M. S. Rahman, S. Nakano, and T. Nishizeki. Box-rectangular drawings of plane graph. In Proceedings of the 21st Graph-Theoretic Concepts in Computer Science, Lecture Notes in Computer Science 1272, pages 250-261. Springer-Verlag, 1999.

32. W. Schnyder. Embedding planar graphs on the grid. In Proceedings of the First Annual ACM-SIAM Symposium on Discrete Algorithms, pages 138-148, 1990.

33. N. Sherwani. Algorithms for VLSI physical design automation. Kluwer Academic Publishers, 1995.

34. L. Stockmeyer. Optimal orientation of cells in slicing floorplan designs. Information and Control, 57(2):91-101, 1983.

35. R. Tamassia. On embedding a graph in the grid with the minimum number of bends. SIAM Journal on Computing, 16(3):421-444, 1987.

36. S. Tsukiyama, K. Koike, and I. Shirakawa. An algorithm to eliminate all complex triangles in a maximal planar graph for use in VLSI floorplan. In Proceedings of the IEEE International Symposium on Circuits and Systems, pages 321-324, 1986.

37. K.-H. Yeap and M. Sarrafzadeh. Floor-planning by graph dualization: 2-concave rectilinear modules. SIAM Journal on Computing, 22(3):500-526, 1993. 